\title{
Frequency synchronization for OFDM Systems Over Doubly-Selective Channels
}

\author{
Jianwu Chen, Yik-Chung Wu and Tung-Sang Ng \\ Department of Electrical and Electronic Engineering \\ The University of Hong Kong, Hong Kong \\ Email: \{jwchen, ycwu, tsng\}@eee.hku.hk
}

\begin{abstract}
In this paper, we investigate the problem of carrier frequency offset (CFO) estimation for Orthogonal frequency division multiplexing (OFDM) system over doubly-selective channels. Representing the doubly-selective channels with basis expansion, the signal model is reformulated and one CFO estimator is derived. Furthermore, the Cramér-Rao bound (CRB) for the estimation problem is derived in closed form. The effectiveness of the proposed scheme is verified by simulations.
\end{abstract}

\section{INTRODUCTION}

Orthogonal frequency division multiplexing (OFDM) systems are sensitive to frequency synchronization errors. The mismatch of the local oscillators at transceivers results in carrier frequency offset (CFO). Doppler spread, which is due to changing channel conditions between the transmitter and the receiver, also contributes to the frequency offset. In the presence of CFO, the orthogonality between subcarriers is destroyed. Then the induced inter-carrier interference (ICI) will cause significant performance degradation. Thus it is critical to estimate the $\mathrm{CFO}$ at the receiver and compensate its effects.

Many methods have been previously proposed to estimate the CFO in OFDM systems. Some of them rely on special pilots (e.g., the repetitive pilots) [1], [2], while the others are based on the inherent structure of OFDM signals (e.g. redundancy in the cyclic prefix (CP) [3]). However, all these CFO estimation schemes are based on an assumption that the channel remains constant during the estimation period. If this assumption is not valid (e.g., in time varying channel), these CFO estimators may suffer great performance degradations or even completely fail. In the literature, there are only few works addressing the problem of CFO estimation in fast fading channel [4], [5]. In [4], two maximum likelihood (ML) approaches, one in frequency-domain and the other one in time-domain, are proposed. In both schemes, the estimators are constructed by extracting the phase difference between two identical OFDM blocks. Unfortunately, since the assumptions and approximations made in these two schemes are only valid for a small Doppler spread in the channel, they are not suitable for fast fading systems. In [5], a simple and computationally attractive Pulse-Pair (PP) method is proposed. However, since this estimator is based on calculating the autocorrelation sequence and thus require a large number of observations, it is not suitable for packet-based transmission.

In this paper, the CFO estimation problem for packet-based
OFDM systems in fast fading channels is investigated. The contributions of this paper are as follows. Focusing on the preamble based structures of many OFDM systems, we first formulate the estimation problem based on one OFDM training block. It is found that the large number of unknown channel coefficients in the signal model render the direct estimation of CFO impossible. Then, the Basis Expansion Model (BEM) is exploited to reformulate the problem and a CFO estimator is derived under Bayesian framework. Furthermore, to verify the efficiency of the proposed estimator, the Cramér-Rao bound $(\mathrm{CRB})$ is derived in closed form.

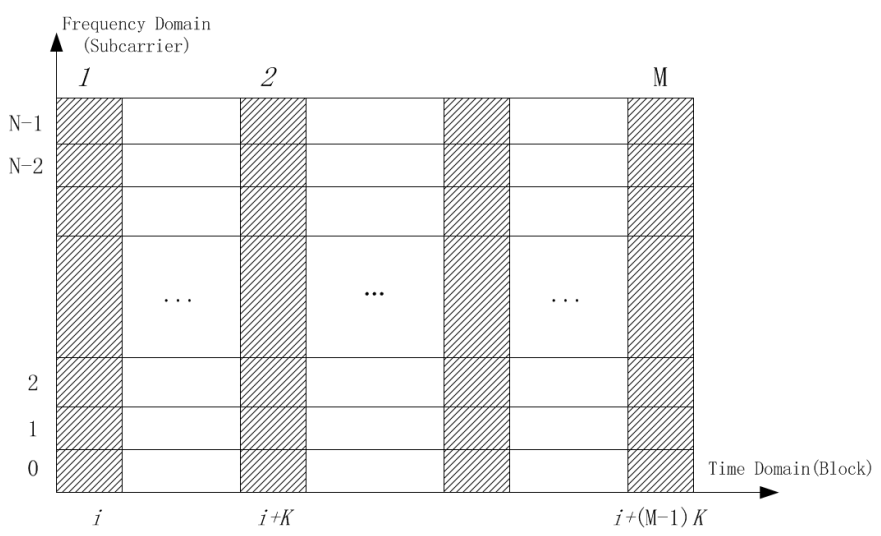

Fig. 1. Packet structure for the considered OFDM system

\section{SySTEM MODEL}

Since the preamble based OFDM system has been adopted in many wireless standards [6], [7], in the following, a preamble-based OFDM system with $N$ subcarriers (as shown in Fig. 1) is considered. In the preamble section, the pilots occupy all subcarriers and after each preamble, one data section consist of $K$ OFDM blocks follows. At the transmitter, the frequency domain signal in $i$ th OFDM block $\mathbf{d}^{(i)}=\left[d^{(i)}(0), d^{(i)}(1), \ldots, d^{(i)}(N-1)\right]^{T}$ is first modulated onto different subcarriers. The corresponding time-domain vector $\mathbf{s}^{(i)}=\left[s^{(i)}(0), s^{(i)}(1), \ldots, s^{(i)}(N-1)\right]^{T}$ is given by $\mathbf{s}^{(i)}=\mathbf{F}^{H} \mathbf{d}^{(i)}$, where $\mathbf{F}$ is the FFT matrix with $\mathbf{F}(k, l)=$ $\frac{1}{\sqrt{N}} e^{-\frac{\jmath 2 \pi k l}{N}}, k, l \in 0, \ldots, N-1$. A cyclic prefix (CP) of length $L_{c p}$ is inserted ahead of $\mathbf{s}^{(i)}$ to cope with the inter-symbol 
interference (ISI) caused by multipath fading. The time varying channel impulse response (including all transmit/receive filtering) $h(l, n)$ is not only a function of multi-path delay tap $l$, but also a function of time index $n$. The normalized CFO between the transmitter and receiver is denoted by $\varepsilon_{o}$. At the receiver, after $\mathrm{CP}$ removal, the received signal vector $\mathbf{x}$, which consists of $N$ consecutive samples, is given by

$$
\mathbf{x}^{(i)}=\boldsymbol{\Gamma}^{(i)}\left(\omega_{o}\right) \mathbf{H} \mathbf{s}^{(i)}+\mathbf{v}^{(i)}
$$

where

$$
\begin{gathered}
\omega_{o} \triangleq 2 \pi \varepsilon_{o} / N, \\
\Gamma^{(i)}\left(\omega_{o}\right)=e^{\jmath\left(i\left(N+L_{c p}\right)\right) \omega_{o}} \cdot \operatorname{diag}\left(1, \ldots, e^{\left(\jmath(N-1) \omega_{o}\right)}\right), \\
\mathbf{v}^{(i)} \triangleq\left[v^{(i)}(0), \ldots, v^{(i)}(N-1)\right]^{T} .
\end{gathered}
$$

$\mathbf{v}^{(i)}$ denotes the complex white Gaussian noise with zero mean and covariance matrix $C_{v}=E\left\{\mathbf{v}^{(i)}\left(\mathbf{v}^{(i)}\right)^{H}\right\}=\sigma^{2} \mathbf{I}_{N}$. $\mathbf{H}$ denotes the doubly-selective channel matrix, which is constructed as

$\mathbf{H}(n,:)=\operatorname{shift}([h(0, n) 0 \ldots 0 h(L-1, n) \ldots h(1, n)], n)$.

where $\operatorname{shift}(\mathbf{a}, n)$ denotes the $n$ position right circular shift operation on the vector $\mathbf{a}$.

\section{CFO Estimation IN FAST FADing ChannEL}

\section{A. Channel Modeling by CE-BEM}

In the signal model (1), there are a large number of unknown coefficients in the matrix $\mathbf{H}$ due to the time-varying channel. Thus, the CFO $\omega_{o}$ can not be estimated directly from (1). To reduce the number of unknowns, basis expansion models have gained popularity recently [8], [9], [10], [11]. In [8], [9], [10], [11], a complex exponential (CE) BEM is employed to represent the time varying channel. The generalized CE-BEM (GCE-BEM) is expressed as

$$
h(l, n)=\sum_{q=-Q}^{Q} h_{l}(q) e^{j \frac{2 \pi q}{N G} n} \quad n=0, \ldots, N-1
$$

where $h_{l}(q), q \in[-Q, Q]$ is the channel parameters for $l$ th channel tap $(l \in[0, L-1]) ; Q=\left\lceil G N T_{s} f_{D}\right\rceil ;\lceil\cdot\rceil$ stands for the ceiling integer; $T_{s}$ is the sampling duration; $f_{D}$ is the doppler spread; $G$ is an over-sampling ratio in doppler spectrum.

From above, for each channel tap, $h(l, n)$ can be represented by $2 Q+1$ coefficients $h_{l}(q)$. Thus, to fully characterize the elements in $\mathbf{H},(2 Q+1) L$ unknown coefficients are needed.

\section{B. CFO Estimator}

From (1), the received signal $x^{(i)}(n)$ can be expressed as

$x^{(i)}(n)=e^{\jmath\left(i\left(N+L_{c p}\right)+n\right) \omega_{o}} \sum_{l=0}^{L-1} h(l, n) s^{(i)}\left([n-l]_{N}\right)+v^{(i)}(n)$

where $[n-l]_{N}$ denotes the modulo operation between $(n-l)$ and $N$. Substituting (6) into the signal model (7) and after some manipulations, the received signal in (1) can be rewritten as [11]

$$
\mathbf{x}^{(i)}=\boldsymbol{\Gamma}^{(i)}\left(\omega_{o}\right) \mathbf{D}^{(i)} \mathbf{h}_{B E M}+\mathbf{v}^{(i)}
$$

where

$$
\mathbf{h}_{B E M}=\left[\mathbf{h}_{-Q}^{T}, \ldots, \mathbf{h}_{Q}^{T}\right]^{T}
$$

$$
\mathbf{h}_{q}=\left[h_{0}(q), \ldots, h_{L-1}(q)\right]^{T}
$$

$$
\mathbf{D}^{(i)}=\left[\boldsymbol{\Phi}^{(i)}(-Q), \ldots, \boldsymbol{\Phi}^{(i)}(Q)\right]
$$

$$
\boldsymbol{\Phi}^{(i)}(q)=e^{\left(\jmath 2 \pi i\left(N+L_{c p}\right) q / N G\right)} \operatorname{diag}\left(1, \ldots, e^{(j 2 \pi(N-1) q / N G)}\right) \mathbf{B}
$$

$$
\begin{gathered}
\mathbf{B}= \\
{\left[\begin{array}{cccc}
s^{(i)}(0) & s^{(i)}(N-1) & \ldots & s^{(i)}(N-L+1) \\
\vdots & \ddots & s^{(i)}(N-1) & \vdots \\
\vdots & \vdots & \ddots & s^{(i)}(N-1) \\
s^{(i)}(L) & \vdots & \vdots & s^{(i)}(0) \\
\vdots & s^{(i)}(L) & \vdots & \vdots \\
\vdots & \vdots & \vdots & \vdots \\
s^{(i)}(N-1) & s^{(i)}(N-2) & \cdots & s^{(i)}(N-L)
\end{array}\right]}
\end{gathered}
$$

Assuming the channel parameters $\mathbf{h}_{B E M}$ remain constant in the $i \rightarrow i+(M-1) K$-th blocks, the received $M$ training blocks $\mathbf{x}^{(i)}, \mathbf{x}^{(i+K)}, \ldots, \mathbf{x}^{(i+(M-1) K)}$ can be written as a vector form $\mathrm{x}$ as

$$
\mathbf{x}=\left[\begin{array}{c}
\mathbf{x}^{(i)} \\
\mathbf{x}^{(i+K)} \\
\vdots \\
\mathbf{x}^{(i+(M-1) K)}
\end{array}\right]=\boldsymbol{\Gamma}\left(\omega_{o}\right) \mathbf{D h} \mathbf{h}_{B E M}+\mathbf{v}
$$

where

$$
\begin{gathered}
\boldsymbol{\Gamma}\left(\omega_{o}\right)=\operatorname{diag}\left(\boldsymbol{\Gamma}^{(i)}\left(\omega_{o}\right), \ldots, \boldsymbol{\Gamma}^{(i+(M-1) K)}\left(\omega_{o}\right)\right) \\
\mathbf{D}=\left[\mathbf{D}^{(i)} ; \ldots ; \mathbf{D}^{(i+(M-1) K)}\right] \\
\mathbf{v}=\left[\mathbf{v}^{(0)} ; \ldots ; \mathbf{v}^{((M-1) K)}\right]
\end{gathered}
$$

where MATLAB notation has been used in (16) and (17).

Under Bayesian framework and the assumption that the noise variance $\sigma^{2}$ is known, the posterior density with the observation $\mathbf{x}$ for $\left(\omega, \mathbf{h}_{B E M}\right)$ is

$$
P\left(\omega, \mathbf{h}_{B E M} \mid \mathbf{x}\right) \propto P\left(\mathbf{x} \mid \omega, \mathbf{h}_{B E M}\right) P(\omega) P\left(\mathbf{h}_{B E M}\right) .
$$

Since our main interest here is to estimate the synchronization parameter $\omega$, the unknown channel vector $\mathbf{h}_{B E M}$ is considered as nuisance parameter. By analytically integrating the nuisance parameter with the prior distributions $P(\omega)=$ constant and 
$P\left(\mathbf{h}_{B E M}\right) \propto \exp \left(-\mathbf{h}_{B E M}^{H} \boldsymbol{\eta}^{-1} \mathbf{h}_{B E M}\right)$, the marginal density of $\omega$ is given by

$$
\begin{aligned}
& P(\omega \mid \mathbf{x}) \\
& \propto \int P\left(\mathbf{x} \mid \omega, \mathbf{h}_{B E M}\right) P(\omega) P\left(\mathbf{h}_{B E M}\right) d \mathbf{h}_{B E M}
\end{aligned}
$$

Compared with the function $P\left(\mathbf{h}_{B E M}\right)$, the likelihood distribution function usually has a sharper shape in the integration region. That means the likelihood can be treated as a delta function in the integral. Thus, according to Gauss integrations, we have

$$
\begin{aligned}
P(\omega \mid \mathbf{x}) \propto & \exp \left(-\hat{\mathbf{h}}_{B E M}^{H} \boldsymbol{\eta}^{-1} \hat{\mathbf{h}}_{B E M}\right) \cdot \\
& \exp \left(-\frac{\mathbf{x}^{H} \boldsymbol{\Gamma}(\omega)\left(\mathbf{I}_{N}-\mathbf{D}\left(\mathbf{D}^{H} \mathbf{D}\right)^{-1} \mathbf{D}^{H}\right) \boldsymbol{\Gamma}^{H}(\omega) \mathbf{x}}{\sigma^{2}}\right)
\end{aligned}
$$

where

$$
\hat{\mathbf{h}}_{B E M}=\left(\sigma^{2} \boldsymbol{\eta}^{-1}+\mathbf{D}^{H} \mathbf{D}\right)^{-1} \mathbf{D}^{H} \boldsymbol{\Gamma}^{H}(\omega) \mathbf{x}
$$

is the LMMSE estimate (when $\omega$ is fixed) of $\mathbf{h}_{B E M}$.

With the above marginal density, the MAP estimator can be derived as:

$$
\hat{\omega}=\arg \max _{\tilde{\omega}}\{P(\tilde{\omega} \mid \mathbf{x})\}
$$

\section{CRAMÉR-RAO BOUND}

In order to provide a performance reference to the proposed estimator, the CRB for the CFO estimation is derived in this section. In the derivation of the CRB, the index for the first block $i$ is set as 0 for simplicity. The signal model in (1) can be rewritten as

$$
\mathbf{x}=\left[\begin{array}{c}
\mathbf{x}^{(0)} \\
\mathbf{x}^{(K)} \\
\vdots \\
\mathbf{x}^{((M-1) K)}
\end{array}\right]=\boldsymbol{\Gamma}\left(\omega_{o}\right) \sum_{l=0}^{L-1} \mathbf{S}_{l} \mathbf{h}(l)+\mathbf{v}
$$

where

$$
\begin{aligned}
& \mathbf{S}_{l}=\mathbf{I}_{M} \otimes \\
& \quad \operatorname{diag}(s(N-l), \ldots, s(N-1), s(0), \ldots, s(N-l-1))
\end{aligned}
$$$$
\mathbf{h}(l)=\left[\mathbf{h}^{(0)}(l)^{T} \mathbf{h}^{(K)}(l)^{T} \ldots \mathbf{h}^{((M-1) K)}(l)^{T}\right]^{T}
$$$$
\mathbf{h}^{(i)}(l)=\left[\begin{array}{c}
h\left(l, 0+i\left(N+L_{c p}\right)\right) \\
h\left(l, 1+i\left(N+L_{c p}\right)\right) \\
\vdots \\
h\left(l, N-1+i\left(N+L_{c p}\right)\right)
\end{array}\right] .
$$

Since the different channel taps are uncorrelated, the covariance matrix of the signal $\mathbf{x}$ is derived as

$$
\begin{aligned}
\mathbf{R}_{\mathbf{x}} & =\boldsymbol{\Gamma}\left(\omega_{o}\right)\left(\sum_{l=0}^{L-1} \mathbf{S}_{l} \mathcal{E}\left(\mathbf{h}(l) \mathbf{h}^{H}(l)\right) \mathbf{S}_{l}^{H}\right) \boldsymbol{\Gamma}^{H}\left(\omega_{o}\right)+\sigma^{2} \mathbf{I}_{N M} \\
& =\boldsymbol{\Gamma}\left(\omega_{o}\right)\left(\sum_{l=0}^{L-1} \mathbf{S}_{l} \mathbf{R}_{h}(l) \mathbf{S}_{l}^{H}\right) \boldsymbol{\Gamma}^{H}\left(\omega_{o}\right)+\sigma^{2} \mathbf{I}_{N M}
\end{aligned}
$$

where $\mathbf{R}_{h}(l)$ is the covariance matrix for the $l$ th channel tap. Its elements are defined as $\mathcal{E}\left\{h\left(l, n_{i}\right) h^{*}\left(l, n_{j}\right)\right\}=$ $\sigma_{l}^{2} J_{0}\left(2 \pi\left|n_{i}-n_{j}\right| f_{D} T_{s}\right)$, where $\sigma_{l}^{2}=\mathcal{E}_{n}\left\{|h(l, n)|^{2}\right\}$ is the averaged power of the $l$ th tap; $J_{0}(\cdot)$ is the zeroth-order Bessel function of the first kind.

Through some similar derivations as [12], the CRB for the $\mathrm{CFO}$ estimation is given by

$$
\begin{aligned}
& \operatorname{CRB}(\omega)=\left\{\operatorname{tr}\left(\mathbf{R}_{\mathbf{x}}^{-1} \frac{\partial \mathbf{R}_{\mathbf{x}}}{\partial \omega} \mathbf{R}_{\mathbf{x}}^{-1} \frac{\partial \mathbf{R}_{\mathbf{x}}}{\partial \omega}\right)\right\}^{-1} \\
& =\left\{2 \operatorname{tr}\left(\mathbf{R}^{-1} \mathbf{W} \mathbf{R}_{c} \mathbf{R}^{-1} \mathbf{R}_{c} \mathbf{W}-\mathbf{R}^{-1} \mathbf{W} \mathbf{R}_{c} \mathbf{R}^{-1} \mathbf{W} \mathbf{R}_{c}\right)\right\}^{-1}
\end{aligned}
$$

where $\mathbf{W}=\operatorname{diag}\left(\mathbf{W}_{0}, \ldots, \mathbf{W}_{M-1}\right)$,

$$
\mathbf{R}=\mathbf{R}_{c}+\sigma^{2} \mathbf{I}_{N M},
$$

$$
\begin{gathered}
\mathbf{W}_{p}=\left[p\left(N+L_{c p}\right), \ldots, p\left(N+L_{c p}\right)+N-1\right] \\
\mathbf{R}_{c}=\sum_{l=0}^{L-1} \mathbf{S}_{l} \mathbf{R}_{h}(l) \mathbf{S}_{l}^{H}
\end{gathered}
$$

and tr denotes trace operator.

\section{Simulations}

\section{A. Parameter Setting}

In the simulations, the considered OFDM system has the following parameters unless stated otherwise:

- OFDM Symbol Size : $N=64$

- Length of CP : $L_{c p}=16$

- Length of channel : $L=8$

- Number of data blocks between two training blocks: $K=$ 3

- Carrier Frequency : $f_{c}=2 \mathrm{GHz}$

- Sampling time : $T_{s}=\frac{1}{f_{s}}=7.0312 \times 10^{-6} \mathrm{~s}$

- Speed of the Vehicle : $v=120 \mathrm{~km} / \mathrm{h}$

- Doppler Spread : $f_{D}=\frac{v f_{c}}{c}=222 \mathrm{~Hz}$

All channel taps are assumed to have the same normalized Doppler Spread $f_{D} N T_{s}=0.1$ and each tap is generated by Jakes model independently. The generalized BEM in (6) is exploited in the channel modeling and the over-sampling ratio is set as $G=10$. The pilot is constructed by a Chu-sequence [13]. The exponential power delay profile is assumed for the multipath fading channel. In each run, the normalized CFO $\varepsilon_{o}$ is generated as a random variable uniformly distributed in $[-0.5,0.5]$. All results are averaged over 5000 Monte Carlo runs.

\section{B. Efficiency of the Proposed Estimation Scheme}

To show the effectiveness of the proposed scheme, the mean square error (MSE) performance of the CFO estimator is plotted versus signal-to-noise ratio (SNR) in Fig. 2, where only one OFDM block is used as training $(M=1)$. The CRB is also shown as reference. For the proposed CFO estimator, the MSE always coincides with the $\mathrm{CRB}$ which means that the proposed estimation scheme is efficient. To emphasize the superiority of 
the proposed CFO estimator, the performance of the schemes in [1] and [4] are also plotted for comparisons. For the scheme in [1], which is proposed for static channel, the simulation shows that this estimator has poor performance over the whole SNR range. An extension of this estimator is proposed in [4] to cope with time varying channels. However, it can be seen that in the fast fading channel, the estimator in [4] can only improve the performance slightly. On the contrary, it is noted that the proposed CFO estimator significantly outperforms the existing methods.

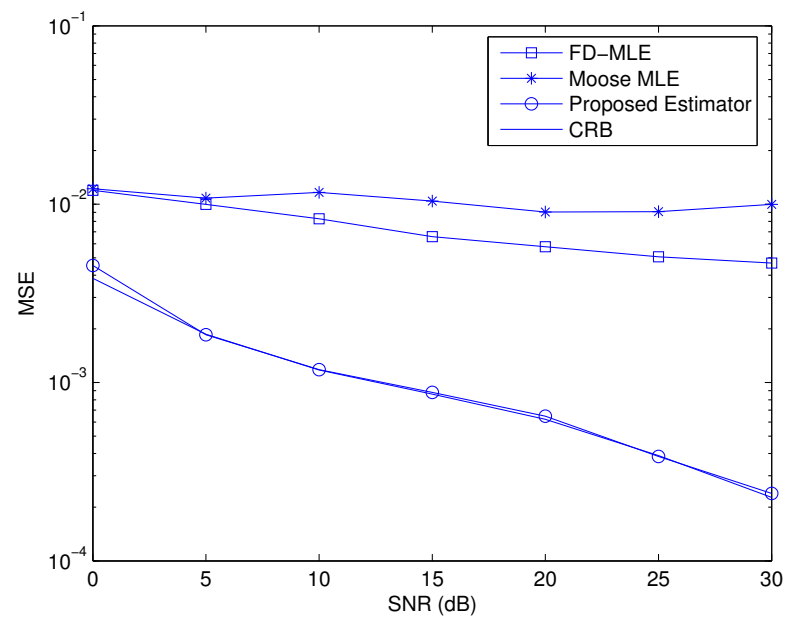

Fig. 2. Performance comparison of various CFO estimation schemes in fast fading channel

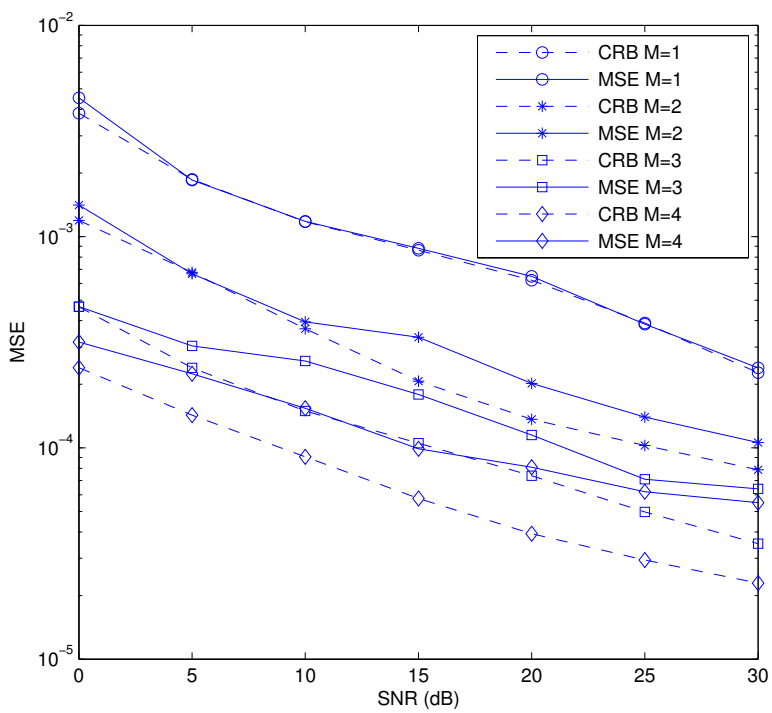

Fig. 3. Performance comparison of different training block length $M$

\section{Effect of the Number of Training blocks}

In Fig. 3, the MSE performances of the proposed CFO estimator with different training block lengths $(M)$ are plotted.
It can be seen that if multiple OFDM blocks $(M)$ are exploited in the CFO estimation, the estimation performance can be improved. However, it should also be noted that when more than one training block is used in the estimation, the MSE performance will depart from the corresponding CRBs. This is because the assumption that the BEM expansion coefficients are constant in these blocks may not be valid in this case.

\section{CONCLUSION}

In this paper, the problem of CFO estimation for OFDM system over doubly-selective channels has been considered. Based on the BEM for doubly-selective channels, the received signal model is rewritten and one CFO estimator is derived under Bayesian framework. To evaluate the performance of the proposed estimator, the CRB for the estimation problem has also been derived in closed form. Simulation results have clearly verified the effectiveness of the proposed scheme.

\section{ACKNOWLEDGMENT}

This work was supported by a grant, CERG HKU 7160/06E.

\section{REFERENCES}

[1] P. H. Moose, "A technique for orthogonal frequency division multiplexing frequency offset correction," IEEE Trans. Commun., vol 42, no. 10, pp. 2908-2914, 1994.

[2] T. M. Schmidl and D. C. Cox, "Robust frequency and timing synchronization for OFDM," IEEE Trans. Commun., vol. 45, no. 12, pp. 1613-1621, Dec. 1997.

[3] J.J.van de Beek, M. Sandell, and P. O. Borjesson, "ML estimation of time and frequency offset in OFDM systems," IEEE Trans. Signal Process., vol. 45, no. 7, pp. 1800-1805, Jul. 1997.

[4] H. Zhou, A. V. Malipatil, and Yih-Fang Huang, "Maximum-likelihood carrier frequency offset estimation for OFDM systems in fading channels," Proceedings of the IEEE Wireless Communications and Networking Conference (WCNC 2006), pp. 1461-1464, Las Vegas, NV, USA, Apr. 2006.

[5] S. S. Abeysekera, "Performance of pulse-pair method of doppler estimation," IEEE Trans. Aerosp. Electron. Syst., vol. 34, no. 2, pp. 520-531, Apr. 1998.

[6] IEEE std 802.11a, supplement to part 11: Wireless lan medium access control (mac) and physical layer (phy) specifications: High-speed physical layer in the $5 \mathrm{ghz}$ band. IEEE Std. 802.11a-1999, 1999.

[7] BRAN; HIPERLAN type 2; physical (phy) layer specification (2nd ed.). Technical Specification 101 475. [Online] Available: http://www.etsi.org, 2001.

[8] X. Ma, G. Giannakis, and S. Ohno, "Optimal training for block transmissions over doubly-selective fading channels," IEEE Trans. Signal Process., vol. 51, no. 5, pp. 1351-1366, May 2003.

[9] X. Ma and G. Giannakis, "Maximum-diversity transmissions over doubly selective wireless channels," IEEE Trans. Inform. Theory, vol. 49, no. 7, pp. 1832-1840, Jul. 2003.

[10] X. Ma, G. Giannakis, and B. Lu, "Block differential encoding for rapidly fading channels," IEEE Trans. Commun., vol. 52, no. 1, pp. 416-425, Jan. 2004.

[11] T. Cui, C. Tellambura, and Y. Wu, "Low-complexity pilot-aided channel estimation for OFDM systems over doubly-selective channels," Proceedings of the IEEE International Conference on Communications (ICC), vol. 3, pp. 1980-1984, Seoul, Korea, May 2005.

[12] M. Ghogho, A. Swami, and T. Durrani, "Frequency estimation in the presence of Doppler spread: performance analysis," IEEE Trans. Signal Process., vol. 49, no. 4, pp. 777-789, Apr. 2001.

[13] D. C. Chu, "Polyphase codes with good periodic correlation properties," IEEE Trans. Information Theory, vol. 18, no. 4, pp. 531-532, Jul. 1972. 